\title{
Automatic tracking of medial gastrocnemius fascicle length during human locomotion
}

\author{
Neil J. Cronin, ${ }^{1}$ Christopher P. Carty, ${ }^{1}$ Rod S. Barrett, ${ }^{1}$ and Glen Lichtwark ${ }^{2}$ \\ ${ }^{1}$ Musculoskeletal Research Program, Griffith Health Institute, Griffith University, Queensland; and ${ }^{2}$ School of Human \\ Movement Studies, University of Queensland, Queensland, Australia
}

Submitted 28 April 2011; accepted in final form 8 August 2011

Cronin NJ, Carty CP, Barrett RS, Lichtwark G. Automatic tracking of medial gastrocnemius fascicle length during human locomotion. J Appl Physiol 111: 1491-1496, 2011. First published August 11, 2011; doi:10.1152/japplphysiol.00530.2011._During human locomotion lower extremity muscle-tendon units undergo cyclic length changes that were previously assumed to be representative of muscle fascicle length changes. Measurements in cats and humans have since revealed that muscle fascicle length changes can be uncoupled from those of the muscle-tendon unit. Ultrasonography is frequently used to estimate fascicle length changes during human locomotion. Fascicle length analysis requires time consuming manual methods that are prone to human error and experimenter bias. To bypass these limitations, we have developed an automatic fascicle tracking method based on the Lucas-Kanade optical flow algorithm with an affine optic flow extension. The aims of this study were to compare gastrocnemius fascicle length changes during locomotion using the automated and manual approaches and to determine the repeatability of the automated approach. Ultrasound was used to examine gastrocnemius fascicle lengths in eight participants walking at 4,5,6, and $7 \mathrm{~km} / \mathrm{h}$ and jogging at $7 \mathrm{~km} / \mathrm{h}$ on a treadmill. Ground reaction forces and three dimensional kinematics were recorded simultaneously. The level of agreement between methods and the repeatability of the automated method were quantified using the coefficient of multiple correlation (CMC). Regardless of speed, the level of agreement between methods was high, with overall CMC values of $0.90 \pm 0.09$ (95\% CI: 0.86-0.95). Repeatability of the algorithm was also high, with an overall CMC of $0.88 \pm 0.08$ (95\% CI: 0.79-0.96). The automated fascicle tracking method presented here is a robust, reliable, and time-efficient alternative to the manual analysis of muscle fascicle length during gait.

ultrasonography; affine flow; muscle fascicle; walking; running

LOWER EXTREMITY MUSCLE-TENDON units (MTUs) undergo cyclic length changes during human locomotion. It was previously assumed that MTU length changes were also representative of muscle fiber (or fascicle) length changes, but measurements in cats and humans have revealed that muscle fascicle length changes can be uncoupled from those of the MTU due to the presence of series elasticity $(11-13,15)$. Recent efforts to better understand dynamic muscle-tendon interaction have therefore focused on examining fascicle behavior during functional tasks such as locomotion (19), as well as changes in muscle-tendon interaction that occur in ageing (25), after training (18), and in various clinical conditions such as diabetes $(7)$.

Ultrasonography has frequently been used to estimate muscle fascicle length changes during walking $(11,19,20)$. To

Address for reprint requests and other correspondence: N. J. Cronin, Department of Biology of Physical Activity, Viveca 234, University of Jyväskylä, 40014 Finland (e-mail: neil.j.cronin@jyu.fi). date, analysis of fascicle length during locomotion has been performed manually, where the experimenter digitizes features of the fascicles for each frame of ultrasound data collected during the gait cycle. This approach is time consuming and potentially prone to human error and experimenter bias. To bypass these limitations, attempts have been made to develop an automated approach. To date, automated techniques have been used to estimate tendinous tissue length changes $(5,24)$, contractile component length changes (22), muscle fascicle orientation (28), and muscle fascicle length (26). However, most of these studies were not performed during locomotion and only one specifically tracked fascicle length changes, which was limited to slow ankle movements (26). It therefore remains unknown to what extent automated algorithms can be used to estimate fascicle length changes during human locomotion.

We have implemented an efficient automatic fascicle tracking method based on the Lucas-Kanade optical flow algorithm (23) with an affine optic flow extension (2). The aims of this study were 1 ) to determine the level of agreement between our automated fascicle tracking algorithm and manual tracking during human locomotion and 2) to assess the repeatability of the automated fascicle tracking algorithm. It was hypothesized that the automated method would yield fascicle length estimates that were in close agreement with those of the manual approach and that the fascicle lengths obtained from the automated approach would be highly repeatable.

\section{METHODS}

\section{Participants}

Eight healthy participants ( 7 men, 1 woman; age $30 \pm 6$ yr; height $180 \pm 8 \mathrm{~cm}$; body mass $75 \pm 8 \mathrm{~kg}$ ) with no history of neurological, cognitive, metabolic, cardiovascular, pulmonary, or lower limb musculoskeletal impairment volunteered to participate in this study. Prior to testing, participants were fully informed of the experimental procedures, and each participant provided written informed consent. The study was approved by the institutional human research ethics committee and was performed in accordance with the Declaration of Helsinki.

\section{Protocol}

Participants walked at speeds of $4,5,6$, and $7 \mathrm{~km} / \mathrm{h}$ and jogged at $7 \mathrm{~km} / \mathrm{h}$ on a split-belt treadmill in a randomized order. Prior to data collection participants walked or jogged at each speed for a minimum of $30 \mathrm{~s}$ to facilitate adaptation to the speed and 30-40 s of data were subsequently collected.

\section{Data Collection and Analysis Procedures}

Gait characteristics. Kinematics of the pelvis and right lower extremity were recorded at $100 \mathrm{~Hz}$ using a four-camera three dimen- 
sional (3D) motion analysis system (Vicon, Oxford Metrics; Oxford, UK). Reflective markers were placed in accordance with the lower body model of Besier et al. (4). An additional marker was placed on the ultrasound probe to track vertical displacement of the probe relative to the ankle joint center. Knee and ankle joint angles during each stride were determined from inverse kinematic analysis of the marker trajectories using a lower body model $(9,14)$ in Opensim software Version 2.0 (8). GRFs were recorded separately from each leg at $1 \mathrm{kHz}$ using eight tri-axial force sensors embedded in the treadmill (Bertec, OH). Vertical GRF was used to estimate the step and stride times as well as the peak GRF for each step. At each speed, steps were only included in the analysis if step duration was within $\pm 5 \%$ of the averaged step duration. This resulted in the inclusion of $27 \pm 5$ steps per condition across all speeds. All reported GRF values were expressed relative to their respective peak values during walking at $4 \mathrm{~km} / \mathrm{h}$.

Ultrasound data collection. A personal computer-based ultrasound system (Echoblaster 128; Telemed, Vilnius, Lithuania) and a 96element linear probe (B-mode; $7 \mathrm{MHz} ; 60 \mathrm{~mm}$ field of view) were used to image the medial gastrocnemius muscle fascicles at a sampling frequency of $80 \mathrm{~Hz}$. The probe was first aligned to the mediolateral midline of the muscle at the level of the midbelly and then aligned with the fascicle plane (3) to minimize errors due to probe orientation (17). The probe was secured over the skin surface with a compressive bandage to minimize probe movement relative to the skin. Rotation of the probe was also minimized due to its flat shape, which leads to very little inertia around the sagittal plane axis $\left[\sim 0.8^{\circ}\right.$ rotation in the mediolateral direction (21)]. A digital output signal from the ultrasound system was used to synchronize data collection. All ultrasound data were recorded to file for subsequent offline analysis.

Automated ultrasound analysis. Automated processing was performed in a custom written Matlab graphical user interface (Mathworks). In the first ultrasound image of each video sequence, which corresponded to five frames before ground contact, the examiner selected the muscle region of interest and defined the fascicle endpoints. The muscle region of interest was defined as the area between the superficial and deep aponeuroses of the MG muscle that was visible in the ultrasound image. Muscle fascicle length was defined as the straight line distance between the superficial and the deep aponeuroses parallel to the lines of collagenous tissue visible in the image (see online supplementary video 1 for an example of the automated tracking process). All measurements were made in the middle of the image where the full length of the fascicle could be visualized in the first ultrasound frame. This method of obtaining representative ultrasound images of MG fascicle length has been shown to minimize length measurement errors (3). Fascicle length changes were subsequently tracked using a Lucas-Kanade optical flow algorithm with affine optic flow extension $(2,23)$. The affine optic flow model has six parameters: vxt, optic flow at origin (top left corner) in x-direction; vyt, optic flow at origin (top left corner) in y-direction; d, rate of dilation; r, rate of rotation; s1, shear along the main image axis; s2, shear along the diagonal axis.

These parameters can be used to estimate the flow vector (change in position - vx, vy) at specific points in the image $(\mathrm{x}, \mathrm{y})$ by applying the following first order model (30):

$$
(\mathrm{vx}, \mathrm{vy})=\left[\begin{array}{lll}
\mathrm{x} & \mathrm{t} & 1
\end{array}\right] \times\left[\begin{array}{cc}
\mathrm{d}+\mathrm{s} 1 & \mathrm{~s} 2+\mathrm{r} \\
\mathrm{s} 2-\mathrm{r} & \mathrm{d}-\mathrm{s} 1 \\
\mathrm{vxt} & \mathrm{vyt}
\end{array}\right]
$$

The Matlab algorithm used here (contributed by Dr. David Young and available from Matlab Central; see ACKNOwLEDGMENTS) implements a least squares fit of the above parameters to estimates of the spatial and temporal gray-level gradients on a rectilinear grid within the defined region of interest. To calculate the spatial and temporal gradients, the images are first smoothed in both the $\mathrm{x}$ and $\mathrm{y}$ compo- nents by convoluting the image with a 1D Gaussian mask with an appropriate width. In the implementation used here, the Matlab function "fspecial" is used to create the mask with a width of 2.6 times the parameter "sigma," which is the standard deviation of the distribution (set to 3 pixels for this study). To calculate the spatial gradients, the symmetric local difference of the average of the two smoothed images is calculated by $2 \mathrm{D}$ convolution in the $\mathrm{x}$ and $\mathrm{y}$ directions using a simple linear gradient function. The temporal gradients are calculated as the difference between the two smoothed images. The affine flow parameters are then solved by a least square fit of the parameters to achieve the given spatial and temporal gradients. The $\mathrm{x}$ and $\mathrm{y}$ grids along with the spatial and temporal gradients are then resampled ( 3 pixels width) to reduce the data used in the least squares solution and improve processing time. Only data within the defined region of interest are used to obtain the least squares solution. Once the parameters for the model are determined, the flow at individual points can be determined using Eq. 1. Using the model it is possible to calculate the change in position of any $\mathrm{x}-\mathrm{y}$ point from the template image to the tracking image using the transformation (including those outside of the region of interest).

The calculated affine transformation is applied to the defined fascicle end points from one frame to the next. This iterative approach allows fascicle length to be defined for each ultrasound image in a sequence (see Fig. 1 for a schematic of the workflow for each iteration). Demonstration code of the Matlab implementation can also be found in the supplementary information.

Manual ultrasound analysis. Manual analysis was performed using a Matlab-based frame-by-frame method with a two-point fascicle model (proximal and distal) identical to that employed by the automated algorithm. The three trials that most closely resembled the mean ankle joint range of motion throughout the entire trial were selected for further analysis, and the same three trials were analyzed with both methods. Manual ultrasound analysis was always performed first to minimize the possibility of experimenter bias. To facilitate comparisons between methods, a screenshot of the fascicle defined in frame 1 during manual analysis was used to help define the fascicle endpoints for automated tracking.

Automated algorithm repeatability. To assess the repeatability of the algorithm, one trial was analyzed five times for each participant and each speed. The trials included in the analysis were presented in a random order and this sequence was then repeated five times. This prevented the examiner from remembering the location of the fascicle end-points for a given trial. The criterion used for this analysis was to locate the most prominent fascicle from the middle of the image where the proximal and distal endpoints were visible in frame 1 of the video. All fascicle tracking was performed by the same examiner.

\section{Statistical Analysis}

The coefficient of multiple correlation (CMC) (16) was used to assess the repeatability of the automated tracking algorithm by calculating the overall similarity between the five analyses of a given trial. A modified formulation of the CMC proposed by Ferrari et al. (10) for assessing similarity of waveforms between different methods was used to determine the agreement between automated and manually tracked fascicle data averaged over three gait cycles at each speed. For both methods, similar waveforms return CMC values approaching one, whereas dissimilar waveforms result in $\mathrm{CMC}$ values approaching zero. One-way repeated measures General Linear Model was used to assess the effect of speed $(4,5,6,7 \mathrm{~km} / \mathrm{h}$ walk, $7 \mathrm{~km} / \mathrm{h}$ jog) on all outcome measures. Mauchly's test was used to evaluate the assumption of sphericity. Where this assumption was violated, Geisser-Greenhouse (GG) adjustments were used. The minimum level of statistical significance was set at $P<0.05$. Data are reported as means $\pm \mathrm{SD}$ where appropriate. 


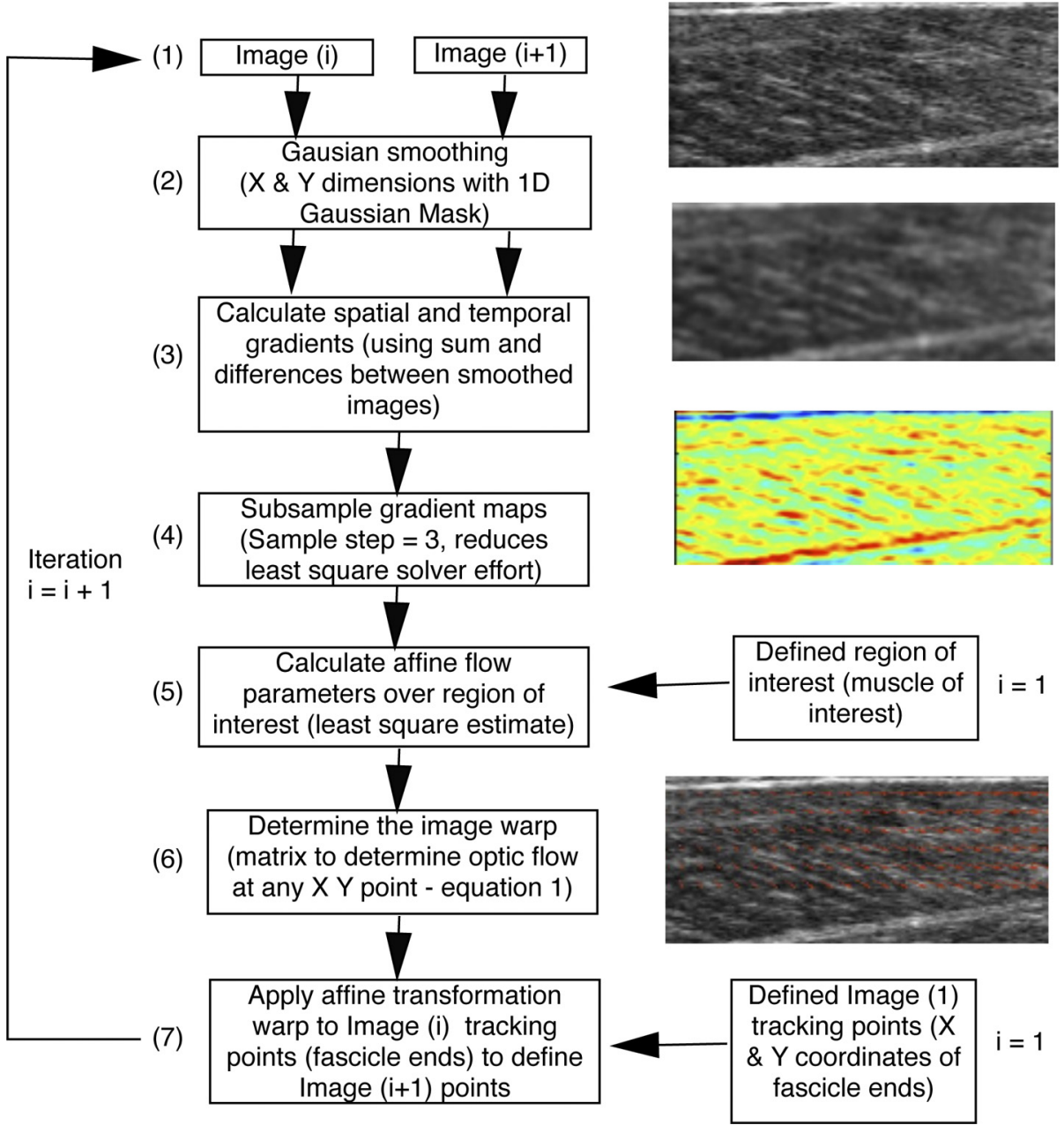

Fig. 1. Workflow of each iteration of the algorithm (frame by frame) used in the present study to track muscle fascicle endpoints.

(3) The diagram includes a graphical representation of the processing involved in each step with numbers to the right of the images corresponding to each step in the workflow. 1, Original image; 2, smoothed image; 3 , spatial gradient in y-direction, represented as a heat map; 6 , calculated flow at different points in images as represented by red vector arrows.

\section{RESULTS}

\section{Gait Characteristics}

As expected there was a tendency for step and stance duration to decrease with increased speed of locomotion, and for ankle and knee range of motion and peak normalized GRF to increase with increased speed of locomotion. In addition, the speed of locomotion did not significantly affect the displacement of the ultrasound probe $\left(F_{2,14} \mathrm{GG}=0.589, P=0.565\right)$.

\section{CMCs}

Fascicle length data from a typical participant throughout an entire step cycle (heel contact to heel contact) at each speed are displayed in Fig. 2A. For this participant the CMCs for comparisons between the automated and manual methods ranged from 0.88 during walking at $7 \mathrm{~km} / \mathrm{h}$ to 0.99 during walking at $4 \mathrm{~km} / \mathrm{h}$. CMC values for repeatability ranged between 0.77 and 0.96 (Fig. 2B). After correction for fascicle length offset between trials, all CMCs for this participant were between 0.98 and 0.99 (Fig. 2C).

Group CMCs for the comparison between the automated and manual approaches and repeatability of the automated algorithm are displayed in Table 1. Mean CMCs for the comparison between approaches ranged from 0.84 during jogging at $7 \mathrm{~km} / \mathrm{h}$ to 0.94 during walking at $4 \mathrm{~km} / \mathrm{h}$. Although there was a tendency for the mean CMCs to decrease with increased locomotion speed, this effect was not statistically significant $\left(F_{2,14}=1.130, P=0.362\right)$. The mean offset in fascicle length between the automated and manual methods was also statistically unaffected by locomotion speed $\left(F_{2,14}=0.388, P=\right.$ 0.815). Mean CMCs for repeatability of the automated algorithm were always at least 0.82 and were not significantly affected by locomotion speed $\left(F_{2,14} \mathrm{GG}=1.813, P=0.372\right)$. After correction for fascicle length offset in the first analysis frame, all CMCs were at least 0.97.

\section{DISCUSSION}

Previous studies of muscle fascicle length changes during locomotion have almost exclusively used manual analysis, which can be highly subjective and time consuming and requires a large number of trials to produce reliable results (1). Irrespective of these limitations manual tracking remains the current "gold standard" for estimating fascicle length changes during locomotion $(6,11,19)$. This study assessed the agreement between a new automated tracking algorithm and the conventional manual approach for tracking medial gastrocnemius fascicle length changes during treadmill locomotion at speeds between 4 and $7 \mathrm{~km} / \mathrm{h}$. The main finding of the study was that the automated and manual fascicle tracking approaches were in close agreement at all locomotion speeds. 
A
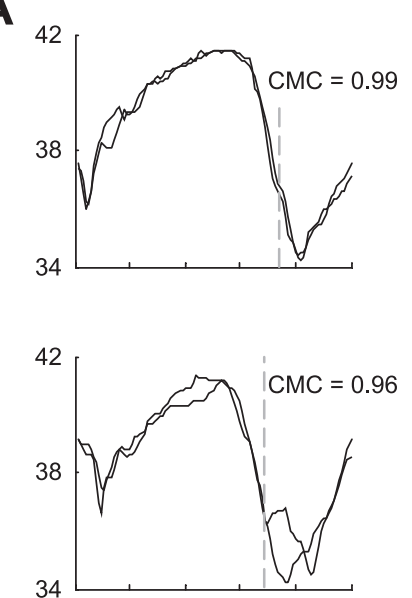
ticipant at all speeds. A: comparison of automated (solid traces) and manual (dashed traces) fascicle tracking. Each trace represents the mean of 3 step cycles. The modified coefficient of multiple correlation (CMC) values calculated using the method of Ferrari et al. (10) are shown for each speed. For this participant, mean fascicle offset between methods across the entire step cycle ranged between $0.26 \pm$ $0.13 \mathrm{~mm}$ during walking at $4 \mathrm{~km} / \mathrm{h}$ and $1.69 \pm$ $0.50 \mathrm{~mm}$ during jogging at $7 \mathrm{~km} / \mathrm{h}$. Dashed vertical lines denote the transition from the stance to swing phase. $B$ : repeatability of the automated tracking algorithm applied to data from the same participant as in $A$. Each panel shows the result of analyzing the same step data 5 times with the tracking algorithm at a single speed. The uncorrected CMC values are shown for each speed. $C$ : the same data as in $B$ after correction for initial differences in fascicle length between trials. The corrected CMC values are also shown.

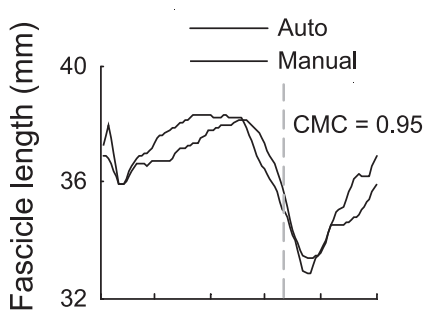

B
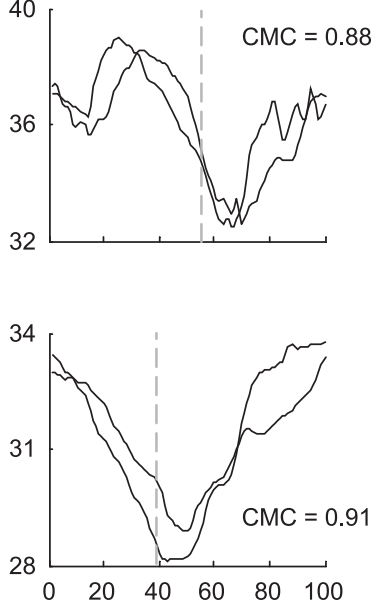

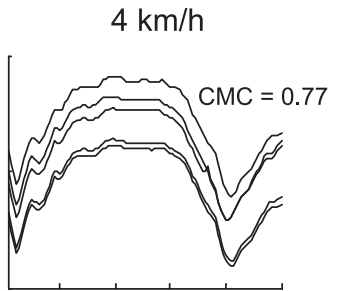

$5 \mathrm{~km} / \mathrm{h}$

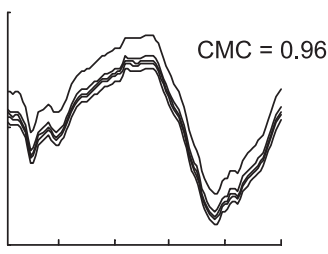

$6 \mathrm{~km} / \mathrm{h}$
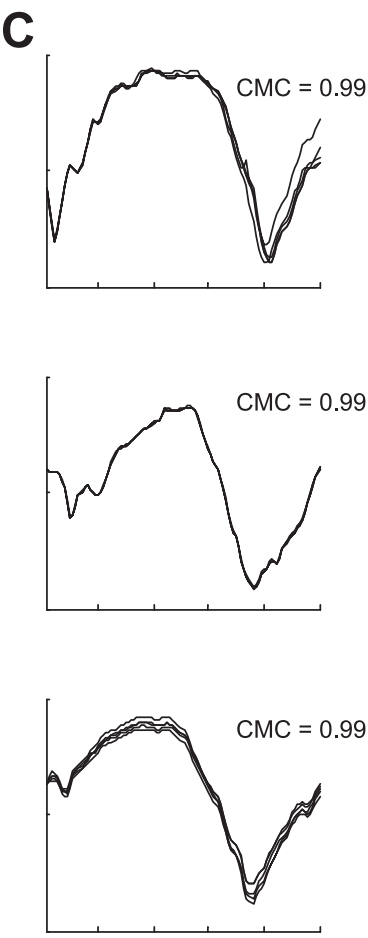

$7 \mathrm{~km} / \mathrm{h}$ walking

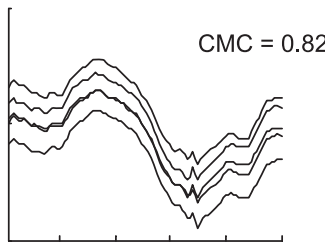

$7 \mathrm{~km} / \mathrm{h}$ jogging

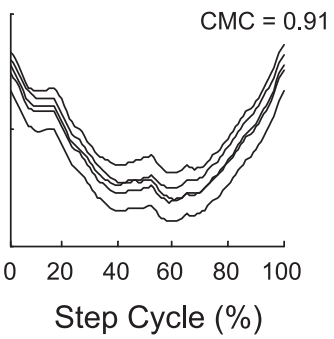

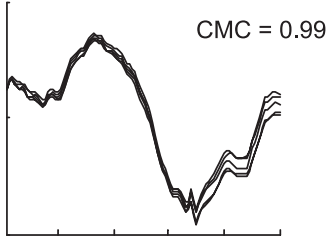

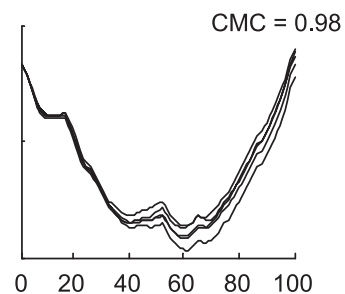

The automated algorithm was also found to be highly repeatable under these conditions. These findings demonstrate that the automated fascicle tracking algorithm provides a viable and efficient means of objectively studying medial gastrocnemius fascicle length changes during human locomotion.

\section{Agreement Between Manual and Automatic Tracking}

There was a high level of agreement between the two methods irrespective of locomotion speed, with an overall CMC computed over all speeds of $0.90 \pm 0.09$ and a corresponding overall $95 \%$ confidence interval from 0.86 to 0.95 . The CMC is a measure of the overall similarity between waveforms, and a CMC value higher than 0.7 indicates excellent similarity (16). This criterion was satisfied at all examined speeds, confirming the suitability of automated tracking as an alternative to manual analysis of fascicle length during locomotion. Importantly, automated tracking is more objective than manual analysis, as it removes the potential for experimenter bias due to knowledge of the expected pattern of fascicle length changes.

\section{Repeatability of the Automated Tracking Algorithm}

Repeatability of the automated tracking algorithm was excellent, with overall CMCs computed from five analyses of the same trial of $0.88 \pm 0.08$ and a $95 \%$ confidence interval of 0.79-0.96. After correction for fascicle offset the mean CMC increased to $0.98 \pm 0.02$. Repeated analyses of the five trials were performed using a simple criterion whereby the most prominent fascicle from the middle of the image was selected. These analyses were also performed in a random order so that 
Table 1. Comparison of automated and manual fascicle tracking and repeatability of the automated algorithm

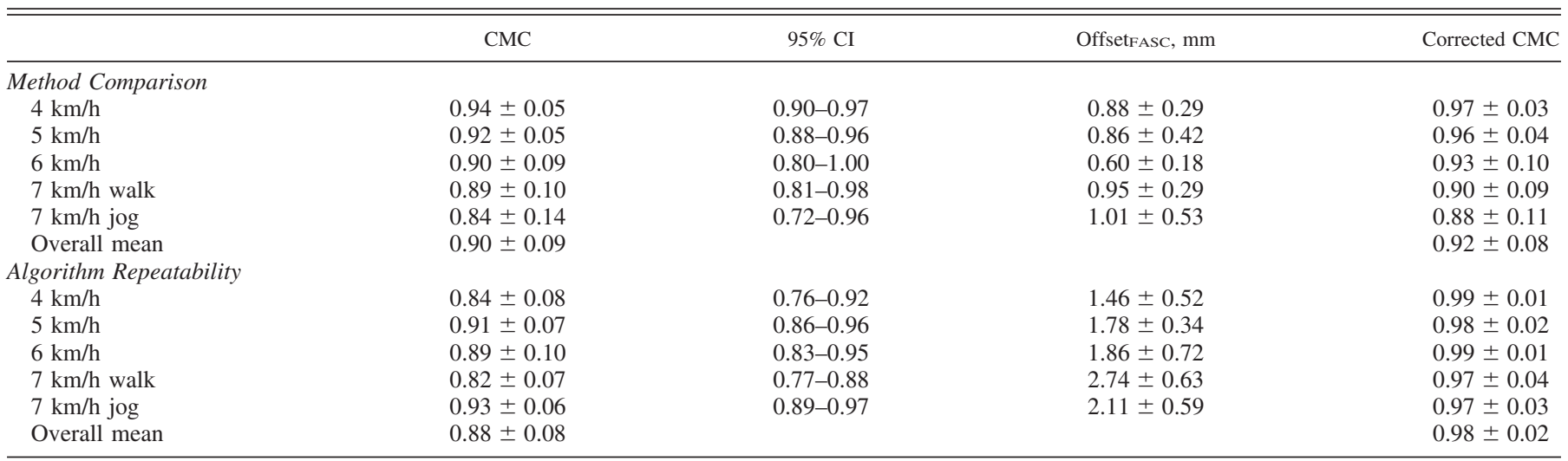

Data are means $\pm \mathrm{SD}, n=8$. OffsetFASC, mean offset in fascicle length between methods (top) or repeated trials (bottom).

visual cues could not be used to analyze precisely the same region of the muscle each time. Despite this restriction, the results of the repeated analyses were still highly repeatable at all examined locomotor speeds and were even greater after correcting for differences in initial fascicle length in the first analysis frame.

\section{Methodological Considerations}

The Lucas-Kanade optical flow algorithm with affine optic flow extension used in the present study offers several important advantages relative to other tracking methods. First, the algorithm uses a measure of global movement rather than the tracking of small regions of interest. The latter method has been used successfully to track low frequency, small amplitude displacements during postural sway (22), but is less successful during gait, where high frequency, relatively large displacements occur. An additional advantage is that automated tracking is generally faster than manual tracking. However, automated tracking does not obviate the need to visually examine the fascicle length estimates produced by the algorithm. Indeed, in $6 \%$ of all trials, individual tracking points were repositioned as visual inspection suggested that the target point had been lost. Given that this tended to occur at higher speeds, the most likely reason for this was the relatively large muscle movements that occurred between successive frames. The stability of the algorithm and its potential application at faster gait speeds may therefore be enhanced by using a higher ultrasound sampling frequency than that used in the present study $(80 \mathrm{~Hz})$.

It is important to note that we make the assumption that the object being tracked (the muscle) conforms to the affine transformations of translation $(\mathrm{x}, \mathrm{y})$, rotation, dilation, shear, and skew. Errors will be introduced if the muscle does not conform to these transformations. However, given the promising results, it seems that this assumption holds true when tracking the planar movement of muscle during gait. Our approach may not be as useful when tracking other movements where local shape changes of the muscle are of interest.

The algorithm was occasionally required to extrapolate fascicle end-points that were not visible in the image in some frames. Although this was not a common occurrence in our data, it is occasionally unavoidable with $2 \mathrm{D}$ ultrasound and relatively short fields of view (3). However, as shown in online supplementary video 2 , the algorithm is able to extrapolate the "missing" points quite successfully, and this process did not produce any obvious differences between the automated and manual results. This is an advantage of this approach over the tracking of local features, which is obviously confined to the area of the image.

An important consideration related to the automated method is that muscle fascicles invariably exhibit curvature during contraction (29). Both methods employed in the present study used a two-point fascicle model whereby the points were placed at each end of the fascicle, thereby failing to account for fascicle curvature. However, curvature is most pronounced at high force levels and short muscle fascicle lengths (27), so it is likely to be a minor factor at the speeds examined here. Visual inspection of our data appeared to confirm this (see online supplementary videos). The algorithm presented here is capable of tracking the global movement of any part of the muscle, so in future versions, it will be possible to track multiple points along a fascicle and thus account for curvature.

\section{Conclusions}

We have presented a robust, repeatable, and time-efficient method of automatically tracking muscle fascicle length changes during gait. The results obtained using automated tracking were in close agreement with those obtained using manual tracking, which currently represents the "gold standard" of fascicle length analysis. The automated method presented here provides a viable alternative to the manual approach for estimating fascicle length changes during human locomotion and has the potential for widespread application in movement sciences more generally.

\section{ACKNOWLEDGMENTS}

The authors acknowledge Dr. David Young (University of Sussex, UK) for developing the Matlab code for the affine optic flow model that was used in this research (http://www.mathworks.com/matlabcentral/fileexchange/27093affine-optic-flow) and for correspondence regarding its implementation.

\section{GRANTS}

G. Lichtwark was supported by a NHMRC Peter Doherty Postdoctoral Fellowship during the development of algorithms and software. N. Cronin was supported by a New Researcher Grant from Griffith University.

\section{DISCLOSURES}

No conflicts of interest, financial or otherwise, are declared by the authors. 


\section{REFERENCES}

1. Aggeloussis N, Giannakou E, Albracht K, Arampatzis A. Reproducibility of fascicle length and pennation angle of gastrocnemius medialis in human gait in vivo. Gait Posture 31: 73-77, 2010.

2. Baker S, Matthews I. Lucas-Kanade 20 years on: A unifying framework. Int J Comp Vis 56: 221-255, 2004

3. Benard MR, Becher JG, Harlaar J, Huijing PA, Jaspers RT. Anatomical information is needed in ultrasound imaging of muscle to avoid potentially substantial errors in measurement of muscle geometry. Muscle Nerve 39: 652-665, 2009.

4. Besier TF, Sturnieks DL, Alderson JA, Lloyd DG. Repeatability of gait data using a functional hip joint centre and a mean helical knee axis. $J$ Biomech 36: 1159-1168, 2003.

5. Bojsen-Moller J, Hansen P, Aagaard P, Svantesson U, Kjaer M, Magnusson SP. Differential displacement of the human soleus and medial gastrocnemius aponeuroses during isometric plantar flexor contractions in vivo. J Appl Physiol 97: 1908-1914, 2004.

6. Cronin NJ, af Klint R, Grey MJ, Sinkjaer T. Ultrasonography as a tool to study afferent feedback from the muscle-tendon complex during human walking. J Electromyogr Kinesiol 21: 197-207, 2011.

7. Cronin NJ, Peltonen J, Ishikawa M, Komi PV, Avela J, Sinkjaer T, Voigt M. Achilles tendon length changes during walking in long-term diabetes patients. Clin Biomech 25: 476-482, 2010.

8. Delp SL, Anderson FC, Arnold AS, Loan P, Habib A, John CT, Guendelman E, Thelen DG. OpenSim: open-source software to create and analyze dynamic simulations of movement. IEEE Trans Biomed Eng 54: 1940-1950, 2007.

9. Delp SL, Loan JP, Hoy MG, Zajac FE, Topp EL, Rosen JM. An interactive graphics-based model of the lower extremity to study orthopaedic surgical procedures. IEEE Trans Biomed Eng 37: 757-767, 1990.

10. Ferrari A, Cutti AG, Cappello A. A new formulation of the coefficient of multiple correlation to assess the similarity of waveforms measured synchronously by different motion analysis protocols. Gait Posture 31: 540-542, 2010.

11. Fukunaga T, Kubo K, Kawakami Y, Fukashiro S, Kanehisa $\mathbf{H}$, Maganaris CN. In vivo behaviour of human muscle tendon during walking. Proc Biol Sci 268: 229-233, 2001.

12. Griffiths RI. Shortening of muscle fibres during stretch of the active cat medial gastrocnemius muscle: the role of tendon compliance. $J$ Physiol 436: 219-236, 1991.

13. Griffiths RI. Ultrasound transit time gives direct measurement of muscle fibre length in vivo. J Neurosci Methods 21: 159-165, 1987.

14. Hamner SR, Seth A, Delp SL. Muscle contributions to propulsion and support during running. $J$ Biomech 43: 2709-2716, 2010.

15. Hoffer JA, Caputi AA, Pose IE, Griffiths RI. Roles of muscle activity and load on the relationship between muscle spindle length and whole muscle length in the freely walking cat. Prog Brain Res 80: 75-85; discussion 57-60, 1989 .

16. Kadaba MP, Ramakrishnan HK, Wootten ME, Gainey J, Gorton G, Cochran GV. Repeatability of kinematic, kinetic, and electromyographic data in normal adult gait. J Orthop Res 7: 849-860, 1989.

17. Klimstra M, Dowling J, Durkin JL, MacDonald M. The effect of ultrasound probe orientation on muscle architecture measurement. J Electromyogr Kinesiol 17: 504-514, 2007.

18. Kubo K, Kanehisa H, Fukunaga T. Effects of resistance and stretching training programmes on the viscoelastic properties of human tendon structures in vivo. J Physiol 538: 219-226, 2002.

19. Lichtwark GA, Bougoulias K, Wilson AM. Muscle fascicle and series elastic element length changes along the length of the human gastrocnemius during walking and running. J Biomech 40: 157-164, 2007.

20. Lichtwark GA, Wilson AM. Interactions between the human gastrocnemius muscle and the Achilles tendon during incline, level and decline locomotion. J Exp Biol 209: 4379-4388, 2006.

21. Lichtwark GA, Wilson AM. In vivo mechanical properties of the human Achilles tendon during one-legged hopping. J Exp Biol 208: 4715-4725, 2005.

22. Loram ID, Maganaris CN, Lakie M. Use of ultrasound to make noninvasive in vivo measurement of continuous changes in human muscle contractile length. J Appl Physiol 100: 1311-1323, 2006.

23. Lucas BD, Kanade T. An iterative image registration technique with an application to stero vision. In: Proceedings of the Seventh International Joint Conference on Artificial Intelligence. Vancouver, Canada, 1981.

24. Magnusson SP, Hansen $\mathbf{P}$, Aagaard $\mathbf{P}$, Brond $\mathbf{J}$, Dyhre-Poulsen $\mathbf{P}$, Bojsen-Moller J, Kjaer M. Differential strain patterns of the human gastrocnemius aponeurosis and free tendon, in vivo. Acta Physiol Scand 177: $185-195,2003$

25. Mian OS, Thom JM, Ardigo LP, Minetti AE, Narici MV. Gastrocnemius muscle-tendon behaviour during walking in young and older adults. Acta Physiol (Oxf) 189: 57-65, 2007.

26. Miyoshi T, Kihara T, Koyama H, Yamamoto S, Komeda T. Automatic detection method of muscle fiber movement as revealed by ultrasound images. Med Eng Phys 31: 558-564, 2009.

27. Muramatsu T, Muraoka T, Kawakami Y, Shibayama A, Fukunaga T. In vivo determination of fascicle curvature in contracting human skeletal muscles. J Appl Physiol 92: 129-134, 2002.

28. Rana M, Hamarneh G, Wakeling JM. Automated tracking of muscle fascicle orientation in B-mode ultrasound images. J Biomech 42: 2068 2073, 2009

29. Van Leeuwen JL, Spoor CW. Modelling mechanically stable muscle architectures. Philos Trans R Soc Lond B Biol Sci 1277: 275-292, 1992.

30. Young D. Affine optic flow [Online]. http://www.mathworks.com/ matlabcentral/fileexchange/27093-affine-optic-flow [September 6, 2011]. 